\title{
Additive representation in thin sequences, III: asymptotic formulae
}

\author{
by \\ J. Brüdern (Stuttgart), K. KaWAda (Morioka) and \\ T. D. Wooley (Ann Arbor, MI)
}

1. Introduction. Since the early days of the Hardy-Littlewood method, investigations concerning the exceptional set of integers not represented in a prescribed manner have been pursued, in various additive problems of Waring type, as a means of gaining insight going beyond that available through direct analysis. The conventional aim is to establish that the set of exceptions has density zero amongst all natural numbers, or a large subset thereof. Our objective in this series of papers is to obtain analogous conclusions for the set of exceptions even amongst the elements of a thin polynomial sequence, such as the set of integral squares. In our primary opus [4], we discussed in general terms the philosophy underlying our novel approach to this circle of problems, illustrating our ideas with a detailed investigation of the set of integers in quadratic and cubic polynomial sequences failing to admit a representation as the sum of six cubes of natural numbers. Our secondary opus [5] was devoted to the binary Goldbach problem. We now turn our attention to corresponding problems in which, for the number of representations of a prescribed type, one seeks to establish the validity of the expected asymptotic formula for almost all integers in a given polynomial sequence.

The flavour of the results now available is perhaps best illustrated with a discussion of Waring's problem for cubes, and this necessitates the introduction of some notation. Denote by $R_{s}(n)$ the number of representations of $n$ as the sum of $s$ cubes of positive integers. A heuristic application of

2000 Mathematics Subject Classification: 11P05, 11P55.

Key words and phrases: Waring's problem, exceptional sets, asymptotic formulae.

T. D. Wooley is a Packard Fellow and was supported in part by NSF grant DMS9970440. This paper benefitted from visits of various of the authors to Ann Arbor, Kyoto, Oberwolfach and Stuttgart, and the authors collectively thank these institutions for their hospitality and excellent working conditions. 
the circle method suggests that for $s \geq 4$, one should have the asymptotic formula

$$
R_{s}(n)=\frac{\Gamma(4 / 3)^{s}}{\Gamma(s / 3)} \mathfrak{S}_{s}(n) n^{s / 3-1}+o\left(n^{s / 3-1}\right)
$$

where

$$
\mathfrak{S}_{s}(n)=\sum_{q=1}^{\infty} \sum_{\substack{a=1 \\(a, q)=1}}^{q}\left(\frac{1}{q} \sum_{r=1}^{q} e\left(a r^{3} / q\right)\right)^{s} e(-a n / q),
$$

and $e(z)=\exp (2 \pi i z)$. We remark that when $s \geq 4$, the singular series $\mathfrak{S}_{s}(n)$ is known to satisfy the lower bound $\mathfrak{S}_{s}(n) \gg 1$ (see Theorem 4.5 of Vaughan [19]), and thus the relation (1.1) does indeed constitute an asymptotic formula.

The conjectured formula (1.1) has been established by Hardy and Littlewood [9] for $s \geq 9$, and by Vaughan [17] for $s=8$. Subject to the truth of an unproved hypothesis concerning certain Hasse-Weil $L$-functions, one has sharp estimates for the sixth moment of the cubic Weyl sum due to Hooley [12] (see Hooley [13] and Heath-Brown [10] for the sharpest available conclusions), and these permit the conditional proof of (1.1) in the additional case $s=7$. For smaller values of $s$, this formula is known to hold only in an average sense. When $s$ is a natural number, denote by $\mathcal{E}_{s}(N)$ the number of natural numbers not exceeding $N$ for which (1.1) fails. The above mentioned work of Vaughan [17] shows that for a certain positive number $\delta$, one has $\mathcal{E}_{4}(N) \ll N(\log N)^{-\delta}$, whence the asymptotic formula (1.1) holds for almost all $n$. Indeed, by employing refinements due to Boklan [1], one may show that any $\delta<3$ is permissible. The only available conclusion of which we are aware for thin sequences is due to Brüdern and Watt [6], who demonstrate the validity of the formula (1.1) for $R_{4}(n)$ for almost all integers $n$ in certain short intervals. With additional variables it is reasonable to expect stronger conclusions, but this is largely unexplored territory. By combining use of the sharpest available version of Weyl's inequality with the classical approach leading to the above estimate for $\mathcal{E}_{4}(N)$, it is simple to establish that whenever $\varepsilon>0$, one has $\mathcal{E}_{4+t}(N) \ll N^{1-t / 6}(\log N)^{\varepsilon-3+t / 2}$ for $t=1,2,3$. Plainly, these estimates lack sufficient power to provide any conclusions concerning the validity of (1.1) in quadratic sequences of natural numbers $n$ when $s=6$, or cubic sequences when $s=7$. However, as we demonstrate in Section 2 below, it is possible to show that (1.1) does indeed hold when $s=6$, for almost all square values of $n$, and likewise when $s=7$, for almost all cubic values of $n$.

It is convenient henceforth to describe a polynomial $\phi \in \mathbb{Q}[t]$ as being an integral polynomial if, whenever the parameter $t$ is an integer, then the value $\phi(t)$ is also an integer. Next recall the notation pertaining to the equation 
(1.1). When $\phi$ is an integral polynomial and $\gamma$ is a fixed positive number, we denote by $\mathcal{E}_{s, \phi}(N ; \gamma)$ the number of integers $n$ with $1 \leq n \leq N$ for which $\phi(n)$ is positive, and

$$
\left|R_{s}(\phi(n))-\frac{\Gamma(4 / 3)^{s}}{\Gamma(s / 3)} \mathfrak{S}_{s}(\phi(n)) \phi(n)^{s / 3-1}\right|>\frac{\phi(n)^{s / 3-1}}{(\log n)^{\gamma}} .
$$

Our first conclusion shows that when $s=6$ or 7 , the asymptotic formula (1.1) holds for almost all natural numbers lying in a fixed quadratic sequence.

TheOrem 1.1. Let $\phi$ be an integral quadratic polynomial.

(i) When $\gamma$ and $\delta$ are positive numbers with $\delta<\max \{2-\gamma, 5 / 2-2 \gamma\}$, one has $\mathcal{E}_{6, \phi}(N ; \gamma) \ll N(\log N)^{-\delta}$.

(ii) For any positive numbers $\gamma$ and $\delta$, one has $\mathcal{E}_{7, \phi}(N ; \gamma) \ll N^{2 / 3+\delta}$.

In each case, the implicit constant in Vinogradov's notation may depend on $\phi, \gamma$ and $\delta$.

In order to obtain useful conclusions concerning cubic sequences, our methods require the availability of seven cubes in the representation.

THEOREM 1.2. Let $\Phi$ be an integral cubic polynomial, and suppose that $\gamma$ and $\delta$ are positive numbers with $\delta<2-2 \gamma$. Then $\mathcal{E}_{7, \Phi}(N ; \gamma) \ll N(\log N)^{-\delta}$. Here, the implicit constant in Vinogradov's notation may depend on $\Phi, \gamma$ and $\delta$.

It may be worth pointing out that the conclusions of Theorems 1.1 and 1.2 may also be established in many cases where the polynomials $\phi$ and $\Phi$ depend on $N$. In the cases $\phi(t)=M-t^{2}$, where we take $N=M^{1 / 2}$, and $\Phi(t)=M-t^{3}$, where we take $N=M^{1 / 3}$, for example, the above conclusions may be established with the dependence of the implicit constants on $\phi$ and $\Phi$ suppressed. The energetic reader may also care to verify that a similar argument yields a conclusion resembling Theorem 1.1(i), but with the polynomial $\phi(n)$ now replaced by the cubic polynomial $\phi(t, u)=M-t^{3}-u^{3}$. Thus, with this substitution in mind, and with $N=M^{1 / 3}$, one finds that whenever $\delta<2-\gamma$, the inequality (1.2) holds for at most $O\left(N^{2}(\log N)^{-\delta}\right)$ of the pairs $(t, u)$ with $1 \leq t, u \leq N$.

At this stage, rather than describe in detail the strategy for establishing conclusions of the type typified in Theorems 1.1 and 1.2, we refer the reader to our earlier paper [4] for a lengthy discussion concerning the basic plan of attack on such problems. The relevant ideas are described in Section 2 of the present paper. Suffice it to say that on this occasion, we engineer an exponential sum which encodes information concerning the failure of the asymptotic formula (1.1) within the sequence of integers $n$ of interest (see, for example, the exponential sum $K(\alpha)$ defined in (2.6) below). Mean value estimates involving this exponential sum play a prominent role 
within our application of the Hardy-Littlewood method, and it is crucial to our argument that this sum preserve the arithmetic information concerning the sequence of integers under consideration. Such information is frequently lost, or at least exploited rather inefficiently, in more traditional approaches involving the use of Bessel's inequality.

We have concentrated thus far on sums of cubes, but of course the same ideas are applicable for sums of higher powers, and indeed, in certain circumstances, there is little to be lost when one restricts the integers concerned to be prime numbers.

Theorem 1.3. When $k$ is a positive integer, define $s_{1}(k)$ by

$$
s_{1}(k)= \begin{cases}2^{k} & \text { when } 1 \leq k \leq 5, \\ \frac{7}{8} \cdot 2^{k} & \text { when } k \geq 6 .\end{cases}
$$

Then whenever $\phi$ is an integral polynomial with positive leading coefficient, the expected asymptotic formula for the number of representations of the integer $\phi(n)$ as the sum of $s$ kth powers of primes holds for almost all $n$, provided only that $s \geq s_{1}(k)$.

We remark that when $k \geq 9$ or thereabouts, the work of Ford [7] may be used to reduce the permissible choice for $s_{1}(k)$. Thus, when $k$ is large, one may take $s_{1}(k)=(1+o(1)) k^{2} \log k$ in the conclusion of Theorem 1.3. With an additional variable, of course, one may establish a conclusion in which the expected asymptotic formula holds for all large integers (see, for example, Hua [14]). We omit the proof of Theorem 1.3, noting only that estimates of the form

$$
\int_{0}^{1}\left|\sum_{x \leq P} e\left(\alpha x^{k}\right)\right|^{s_{1}(k)} d \alpha \ll P^{s_{1}(k)-k},
$$

due to Vaughan [17], [18] and Boklan [2], together with well-known estimates for the exponential sum

$$
\sum_{p \leq P} e\left(\alpha p^{k}\right)
$$

in which an arbitrarily large power of $\log P$ is saved over the trivial estimate when $\alpha$ lies on a "minor arc", suffice to establish the desired conclusion through a method paralleling that of the proof of Theorem 1.1 in Section 2.

It transpires that our methods offer great flexibility in their application to the study of exceptional sets in additive number theory. In principle the proofs of Theorems 1.1 and 1.2 might be regarded as providing a model for almost any application of our methods. We provide several further results within the compass of our ideas in Sections 3 and 4, together with brief proofs of these results. As illustrations of what is possible beyond Waring's problem for cubes, we mention at this point two further results (but see Theorem 3.2 
below for further conclusions on sums of mixed powers). First we provide a conclusion which can be considered as a strengthening of Theorem 1.1(i). We first require some notation. Write $R(n)$ for the number of representations of $n$ as the sum of five cubes and a sixth power of positive integers, and write

$$
\mathfrak{S}(n)=\sum_{q=1}^{\infty} \sum_{\substack{a=1 \\(a, q)=1}}^{q} q^{-6}\left(\sum_{r=1}^{q} e\left(a r^{3} / q\right)\right)^{5} \sum_{t=1}^{q} e\left(a t^{6} / q\right) e(-a n / q) .
$$

It is a simple consequence of Vaughan [19], Theorem 4.5 , that $\mathfrak{S}(n) \gg 1$ for each integer $n$. When $\phi$ is an integral polynomial and $\gamma$ is a fixed positive number, denote by $\mathcal{E}_{\phi}^{*}(N ; \gamma)$ the number of integers $n$ with $1 \leq n \leq N$ for which $\phi(n)$ is positive and

$$
\left|R(\phi(n))-\frac{\Gamma(4 / 3)^{5} \Gamma(7 / 6)}{\Gamma(11 / 6)} \mathfrak{S}(\phi(n)) \phi(n)^{5 / 6}\right|>\frac{\phi(n)^{5 / 6}}{(\log n)^{\gamma}} .
$$

THEOREM 1.4. Let $\phi$ denote an integral quadratic polynomial with positive leading coefficient. Suppose that $\gamma$ and $\delta$ are positive numbers with $\gamma+\delta<3 / 2$. Then $\mathcal{E}_{\phi}^{*}(N ; \gamma) \ll N(\log N)^{-\delta}$. Here, the implicit constant in Vinogradov's notation may depend on $\phi, \gamma$ and $\delta$.

In a sense, Theorem 1.4 shows that one may restrict one of the cubes in the representation implicit in Theorem 1.1(i) to be a sixth power, and deduce nonetheless that almost all integers in a fixed quadratic sequence possess the expected number of representations.

Next we consider polynomial sequences represented by sums of $k$ th powers, for larger $k$. When $\phi$ is an integral polynomial with positive leading coefficient, denote by $\widetilde{G}_{\phi}^{+}(k)$ the least number $s$ with the property that, for almost all natural numbers $n$, the expected asymptotic formula holds for the number of representations of $\phi(n)$ in the shape

$$
x_{1}^{k}+\ldots+x_{s}^{k}=\phi(n),
$$

with $x_{i} \in \mathbb{N}(1 \leq i \leq s)$.

TheOREM 1.5. Let $\phi$ be an integral polynomial of degree 1 or 2 with positive leading coefficient. Then for large $k$ one has

$$
\widetilde{G}_{\phi}^{+}(k) \leq \frac{1}{2} k^{2}(\log k+\log \log k+O(1)) .
$$

If, on the other hand, $\phi$ is an integral polynomial of degree $l>2$, then for large $k$ one has

$$
\widetilde{G}_{\phi}^{+}(k) \leq(1-1 / l) k^{2}(\log k+\log \log k+O(1)) .
$$

As we mentioned implicitly following the statement of Theorem 1.3, the expected asymptotic formula for sums of $s k$ th powers was established by Ford [7], provided that $s \geq k^{2}(\log k+\log \log k+O(1))$. When $l=1$, the 
conclusion of Theorem 1.5 follows easily from the methods underlying the latter conclusion via a classical argument. We therefore describe the proof of Theorem 1.5 only in the cases in which $l \geq 2$, and it is in fact the latter situation wherein our new approach plays a crucial role. It is curious that the number of variables required almost always to obtain the expected number of representations in the shape (1.4) is almost the same, in the current state of knowledge, for $\phi$ of degree both 1 and 2 .

Throughout, the letter $\varepsilon$ will denote a sufficiently small positive number. We take $P$ to be the basic parameter, a large real number depending at most on $\varepsilon$ and any coefficients of implicit polynomials if necessary. We use $\ll$ and $\gg$ to denote Vinogradov's well-known notation, implicit constants depending at most on $\varepsilon$ and implicit polynomials. Sometimes we make use of vector notation. For example, the expression $\left(c_{1}, \ldots, c_{t}\right)$ is abbreviated to $\mathbf{c}$. Also we write $[x]$ for the greatest integer not exceeding $x$, and $\lceil x\rceil$ for the least integer $y$ with $y \geq x$. Summations start at 1 unless indicated otherwise. In an effort to simplify our analysis, we adopt the following convention concerning the parameter $\varepsilon$. Whenever $\varepsilon$ appears in a statement, we assert that for each $\varepsilon>0$ the statement holds for sufficiently large values of the main parameter. Note that the "value" of $\varepsilon$ may consequently change from statement to statement, and hence also the dependence of implicit constants on $\varepsilon$.

2. Waring's problem for cubes. The proof of Theorem 1.1 is sufficiently simple that the principles underlying our methods are clearly visible. We therefore discuss this proof in some detail, so as to provide a model for future analyses.

The proof of Theorem 1.1. Let $\phi$ be an integral quadratic polynomial, and let $N$ be a large real number. We consider a fixed positive number $\gamma$. Suppose that $s=6$ or 7 , and define $\mathcal{Z}_{s}(N)$ to be the set of integers $n$ with $N / 2<n \leq N$ for which $\phi(n)>0$ and the inequality (1.2) holds. Our first goal is to show that whenever $\delta$ is a positive number with $\delta<$ $\max \{2-\gamma, 5 / 2-2 \gamma\}$, then one has $\operatorname{card}\left(\mathcal{Z}_{6}(N)\right) \ll_{\phi} N(\log N)^{-\delta}$. Our second goal is to show that for any positive number $\delta$, one has $\operatorname{card}\left(\mathcal{Z}_{7}(N)\right) \ll_{\phi}$ $N^{2 / 3+\delta}$. By summing over dyadic intervals, one then obtains the bounds for $\mathcal{E}_{s, \phi}(N ; \gamma)(s=6,7)$ claimed in the statement of Theorem 1.1.

Note first that if the leading coefficient of $\phi$ is not positive, then the desired conclusion is trivial. We therefore suppose that the leading coefficient of $\phi$ is positive, and define $P=\phi(N)^{1 / 3}$, so that $P \asymp N^{2 / 3}$. Next define

$$
f(\alpha)=\sum_{1 \leq x \leq P} e\left(\alpha x^{3}\right) .
$$


Then by orthogonality, for each integer $n$ with $N / 2<n \leq N$ one has

$$
R_{s}(\phi(n))=\int_{0}^{1} f(\alpha)^{s} e(-\alpha \phi(n)) d \alpha .
$$

Let $\mathfrak{M}$ denote the union of the intervals

$$
\mathfrak{M}(q, a)=\left\{\alpha \in[0,1):|q \alpha-a| \leq P(6 \phi(N))^{-1}\right\},
$$

with $0 \leq a \leq q \leq P / 6$ and $(a, q)=1$. Then, by Theorem 4.4 of Vaughan [19], there is a positive number $\tau$ such that whenever $N / 2<n \leq N$ one has

$$
\begin{aligned}
\int_{\mathfrak{M}} f(\alpha)^{s} e(-\alpha \phi & (n)) d \alpha \\
& =\frac{\Gamma(4 / 3)^{s}}{\Gamma(s / 3)} \mathfrak{S}_{s}(\phi(n)) \phi(n)^{s / 3-1}+O\left(\phi(n)^{s / 3-1-\tau}\right) .
\end{aligned}
$$

Now write $\mathfrak{m}=[0,1) \backslash \mathfrak{M}$. Then for $n \in \mathcal{Z}_{s}(N)$, it follows from (1.2), (2.2) and (2.3) that

$$
\left|\int_{\mathfrak{m}} f(\alpha)^{s} e(-\alpha \phi(n)) d \alpha\right|>\frac{1}{2} \cdot \frac{\phi(n)^{s / 3-1}}{(\log n)^{\gamma}} .
$$

We next define the complex numbers $\eta_{n}=\eta_{n, s}$ by taking $\eta_{n}=0$ for $n \notin \mathcal{Z}_{s}(N)$, and when $n \in \mathcal{Z}_{s}(N)$ by means of the equation

$$
\left|\int_{\mathfrak{m}} f(\alpha)^{s} e(-\alpha \phi(n)) d \alpha\right|=\eta_{n} \int_{\mathfrak{m}} f(\alpha)^{s} e(-\alpha \phi(n)) d \alpha .
$$

In view of (2.4), one obtains

$$
\begin{aligned}
\frac{\phi(N)^{s / 3-1}}{(\log N)^{\gamma}} \operatorname{card}\left(\mathcal{Z}_{s}(N)\right) & \ll \sum_{N / 2<n \leq N} \eta_{n} \int_{\mathfrak{m}} f(\alpha)^{s} e(-\alpha \phi(n)) d \alpha \\
& =\int_{\mathfrak{m}} f(\alpha)^{s} K(-\alpha) d \alpha
\end{aligned}
$$

where

$$
K(\alpha)=\sum_{N / 2<n \leq N} \eta_{n} e(\alpha \phi(n)) .
$$

We now divide into cases.

(i) Suppose that $s=6$. As our first treatment of the integral on the right hand side of (2.5), we apply Hölder's inequality to obtain

$$
\frac{N^{2}}{(\log N)^{\gamma}} \operatorname{card}\left(\mathcal{Z}_{6}(N)\right) \ll I_{1}^{3 / 4} I_{2}^{1 / 4},
$$


where

$$
I_{1}=\int_{\mathfrak{m}}|f(\alpha)|^{8} d \alpha \quad \text { and } \quad I_{2}=\int_{0}^{1}|K(\alpha)|^{4} d \alpha .
$$

The mean value $I_{1}$ is swiftly estimated by reference to Boklan [1], who established the upper bound

$$
I_{1} \ll P^{5}(\log P)^{\varepsilon-3}
$$

for any positive number $\varepsilon$. The integral $I_{2}$, by orthogonality, is bounded above by the number of integral solutions of the equation

$$
\phi\left(n_{1}\right)-\phi\left(n_{2}\right)=\phi\left(n_{3}\right)-\phi\left(n_{4}\right),
$$

with $1 \leq n_{i} \leq N(1 \leq i \leq 4)$. By completing the square in the quadratic polynomial $\phi$, and considering the associated equation $x_{1}^{2}-x_{2}^{2}=x_{3}^{2}-x_{4}^{2}$, it follows from well-known estimates that

$$
I_{2} \ll N^{2} \log N \text {. }
$$

On collecting together (2.7), (2.9) and (2.10), we conclude that

$$
\begin{aligned}
\frac{N^{2}}{(\log N)^{\gamma}} \operatorname{card}\left(\mathcal{Z}_{6}(N)\right) & \ll P^{15 / 4}(\log N)^{\varepsilon-9 / 4} N^{1 / 2}(\log N)^{1 / 4} \\
& \ll N^{3}(\log N)^{\varepsilon-2}
\end{aligned}
$$

whence for any $\varepsilon>0$ one has

$$
\operatorname{card}\left(\mathcal{Z}_{6}(N)\right) \ll N(\log N)^{\varepsilon+\gamma-2} .
$$

As an alternative to the application of Hölder's inequality implicit in (2.7), we may instead apply Schwarz's inequality to (2.5) to obtain

$$
\frac{N^{2}}{(\log N)^{\gamma}} \operatorname{card}\left(\mathcal{Z}_{6}(N)\right) \ll\left(\sup _{\alpha \in \mathfrak{m}}|f(\alpha)|\right) I_{1}^{1 / 2} I_{3}^{1 / 2},
$$

where

$$
I_{3}=\int_{0}^{1}|f(\alpha) K(\alpha)|^{2} d \alpha
$$

By orthogonality, the integral $I_{3}$ is bounded above by the number of integral solutions of the equation

$$
\phi\left(n_{1}\right)-\phi\left(n_{2}\right)=x_{1}^{3}-x_{2}^{3}
$$

with $n_{i} \in \mathcal{Z}_{6}(N)$ and $x_{i} \leq P(i=1,2)$. Consider any solution $\mathbf{n}, \mathbf{x}$ of (2.14) counted by $I_{3}$. When $n_{1}=n_{2}$ one has $x_{1}=x_{2}$, so that the number of diagonal solutions is at most $P \operatorname{card}\left(\mathcal{Z}_{6}(N)\right)$. Since $N$ is large, meanwhile, when $n_{1} \neq n_{2}$ one has $\phi\left(n_{1}\right) \neq \phi\left(n_{2}\right)$, whence $x_{1}^{3} \neq x_{2}^{3}$. But $n_{1}-n_{2}$ is a divisor of $\phi\left(n_{1}\right)-\phi\left(n_{2}\right)$, so that an elementary estimate shows that for each of the $O\left(P^{2}\right)$ possible choices of $x_{1}, x_{2}$ with $x_{1}^{3} \neq x_{2}^{3}$, one has $O\left(P^{\varepsilon}\right)$ choices 
for $n_{1}-n_{2}$. Fixing any one of the latter choices for $n_{1}-n_{2}$, the equation (2.14) uniquely determines $n_{1}+n_{2}$, and hence also each of $n_{1}$ and $n_{2}$. It follows that the number of non-diagonal solutions of (2.14) counted by $I_{3}$ is $O\left(P^{2+\varepsilon}\right)$. Thus we obtain

$$
I_{3} \ll P \operatorname{card}\left(\mathcal{Z}_{6}(N)\right)+P^{2+\varepsilon} .
$$

Finally, we note that on combining the refined estimates of Hall and Tenenbaum [8] for Hooley's $\Delta$-function with the proof of Lemma 1 of Vaughan [17], one obtains

$$
\sup _{\alpha \in \mathfrak{m}}|f(\alpha)| \ll P^{3 / 4}(\log P)^{1 / 4+\varepsilon} .
$$

On collecting together (2.9), (2.15), (2.16) with (2.12), we may conclude that

$$
\frac{N^{2}}{(\log N)^{\gamma}} \operatorname{card}\left(\mathcal{Z}_{6}(N)\right) \ll P^{15 / 4}(\log P)^{\varepsilon-5 / 4}\left(\operatorname{card}\left(\mathcal{Z}_{6}(N)\right)\right)^{1 / 2}+P^{17 / 4+\varepsilon},
$$

whence the relation $P \asymp N^{2 / 3}$ leads to the upper bound

$$
\operatorname{card}\left(\mathcal{Z}_{6}(N)\right) \ll N(\log N)^{\varepsilon+2 \gamma-5 / 2} .
$$

In view of our opening comments, the conclusion of Theorem 1.1(i) follows on combining (2.11) and (2.17), and summing over dyadic intervals.

(ii) Suppose that $s=7$. In this case we apply Schwarz's inequality to (2.5) to obtain

$$
\frac{N^{8 / 3}}{(\log N)^{\gamma}} \operatorname{card}\left(\mathcal{Z}_{7}(N)\right) \ll\left(\sup _{\alpha \in \mathfrak{m}}|f(\alpha)|\right)^{2} I_{1}^{1 / 2} I_{3}^{1 / 2} .
$$

By an argument paralleling that leading to (2.15), one finds on this occasion that

$$
I_{3} \ll P \operatorname{card}\left(\mathcal{Z}_{7}(N)\right)+P^{2+\varepsilon},
$$

whence by $(2.9)$ and $(2.16)$, we deduce that

$$
\frac{N^{8 / 3}}{(\log N)^{\gamma}} \operatorname{card}\left(\mathcal{Z}_{7}(N)\right) \ll P^{9 / 2}(\log P)^{\varepsilon-1}\left(\operatorname{card}\left(\mathcal{Z}_{7}(N)\right)\right)^{1 / 2}+P^{5+\varepsilon} .
$$

The relation $P \asymp N^{2 / 3}$ in this case leads to the estimate

$$
\operatorname{card}\left(\mathcal{Z}_{7}(N)\right) \ll N^{2 / 3+\varepsilon},
$$

and the conclusion of Theorem 1.1(ii) follows on summing over dyadic intervals.

Having prepared the ground with our proof of Theorem 1.1 above, we may be brief in our discussion of the proof of Theorem 1.2.

The proof of Theorem 1.2. We take $\phi$ to be an integral cubic polynomial, and follow the argument of the proof of Theorem 1.1 down to equations (2.5) 
and (2.6). Our goal is now to show that whenever $\delta<2-2 \gamma$, then one has $\operatorname{card}\left(\mathcal{Z}_{7}(N)\right) \ll_{\phi} N(\log N)^{-\delta}$. Moreover, in the current situation one should note that $P \asymp N$. Fixing $s=7$, and applying Schwarz's inequality to (2.5), we obtain

$$
\frac{N^{4}}{(\log N)^{\gamma}} \operatorname{card}\left(\mathcal{Z}_{7}(N)\right) \ll\left(\sup _{\alpha \in \mathfrak{m}}|f(\alpha)|\right)^{2} I_{1}^{1 / 2} I_{3}^{1 / 2},
$$

where $I_{1}$ and $I_{3}$ are defined as in (2.8) and (2.13), though we stress that in (2.13) the implicit polynomial $\phi$ is now cubic. By orthogonality, the integral $I_{3}$ is bounded above by the number of integral solutions of the equation (2.14) with $n_{i} \in \mathcal{Z}_{7}(N)$ and $x_{i} \leq P(i=1,2)$. The left hand side of the latter equation is divisible by the factor $n_{1}-n_{2}$, and the right hand side of the equation is divisible by $x_{1}-x_{2}$. Moreover, the right hand side of the equation is a homogeneous polynomial in $x_{1}, x_{2}$. An inspection of the argument of the proof of Theorem 1.1 of Skinner and Wooley [15] therefore reveals that $I_{3}$ is bounded above by the number of diagonal solutions, together with at most $O\left(N^{11 / 6+\varepsilon}\right)$ non-diagonal solutions. We therefore obtain

$$
I_{3} \ll N \operatorname{card}\left(\mathcal{Z}_{7}(N)\right)+N^{11 / 6+\varepsilon},
$$

whence by $(2.9),(2.16)$ and (2.18), we deduce that

$$
\begin{aligned}
& \frac{N^{4}}{(\log N)^{\gamma}} \operatorname{card}\left(\mathcal{Z}_{7}(N)\right) \\
& \quad \ll P^{3 / 2}(\log P)^{1 / 2+\varepsilon}\left(N \operatorname{card}\left(\mathcal{Z}_{7}(N)\right)+N^{11 / 6+\varepsilon}\right)^{1 / 2}\left(P^{5}(\log P)^{\varepsilon-3}\right)^{1 / 2} .
\end{aligned}
$$

Consequently,

$$
\operatorname{card}\left(\mathcal{Z}_{7}(N)\right) \ll N(\log N)^{\varepsilon+2 \gamma-2},
$$

and the conclusion of Theorem 1.2 follows on summing over dyadic intervals.

3. Sums of mixed powers. In this section we explore exceptional sets in polynomial sequences for additive problems involving mixed powers. Here one is struck by the utility of sharp mean value estimates for mixed sums of powers familiar to aficionados of the circle method. We recall two such estimates in the form of a lemma.

Lemma 3.1. Suppose that $X$ is a large real number. Let $\phi$ be an integral quadratic polynomial with positive leading coefficient. Let $\kappa$ be a fixed positive number and let $\mathcal{A} \subseteq \mathbb{N} \cap\left[1, \kappa X^{1 / 2}\right]$. Define

$$
F_{j}(\alpha)=\sum_{y \leq X^{1 / j}} e\left(\alpha y^{j}\right) \quad \text { and } \quad G(\alpha)=\sum_{n \in \mathcal{A}} e(\alpha \phi(n)) .
$$

Then

$$
\int_{0}^{1}\left|G(\alpha) F_{4}(\alpha)^{2}\right|^{2} d \alpha \ll X(\log X)^{\varepsilon}
$$


and

$$
\int_{0}^{1}\left|G(\alpha) F_{3}(\alpha) F_{6}(\alpha)\right|^{2} d \alpha \ll X(\log X)^{\varepsilon} .
$$

Proof. On considering the underlying diophantine equations, the estimate (3.1) follows from the argument of the proof of Théorème $2^{\prime}(\mathrm{i})$ of Tenenbaum [16] (see $\S 2$, and in particular the estimation of $W$ on p. 235). The estimate (3.2), meanwhile, follows by applying the methods of Hooley [11] and Hall and Tenenbaum [8].

As our opening salvo in this offensive on mixed powers, we establish Theorem 1.4, thereby setting the pattern for our subsequent discussion.

The proof of Theorem 1.4. Let $\phi$ be an integral quadratic polynomial with positive leading coefficient. We consider a fixed positive number $\gamma$, and define $\mathcal{Z}(N)$ to be the set of integers $n$ with $N / 2<n \leq N$ for which the inequality (1.3) holds. We aim to show that whenever $\delta$ is a positive number with $\delta<3 / 2-\gamma$, then one has $\operatorname{card}(\mathcal{Z}(N)) \ll_{\phi} N(\log N)^{-\delta}$, whence the conclusion of Theorem 1.4 follows on summing over dyadic intervals.

Define $P=\phi(N)^{1 / 3}$, let $f(\alpha)$ be defined as in (2.1), and write

$$
g(\alpha)=\sum_{y \leq P^{1 / 2}} e\left(\alpha y^{6}\right)
$$

Then, for each integer $n$ with $N / 2<n \leq N$ one has

$$
R(\phi(n))=\int_{0}^{1} f(\alpha)^{5} g(\alpha) e(-\alpha \phi(n)) d \alpha
$$

Defining the arcs $\mathfrak{M}$ and $\mathfrak{m}$ as in the proof of Theorem 1.1, one may apply the argument of the proof of Theorem 4.4 of Vaughan [19] (with trivial summation over the variable corresponding to the sixth power) to establish that there is a positive number $\tau$ such that whenever $N / 2<n \leq N$, one has

$$
\int_{\mathfrak{M}} f(\alpha)^{5} g(\alpha) e(-\alpha \phi(n)) d \alpha=\frac{\Gamma(4 / 3)^{5} \Gamma(7 / 6)}{\Gamma(11 / 6)} \mathfrak{S}(\phi(n)) \phi(n)^{5 / 6}+O\left(\phi(n)^{5 / 6-\tau}\right) \text {. }
$$

Thus we may conclude that for $n \in \mathcal{Z}(N)$,

$$
\left|\int_{\mathfrak{m}} f(\alpha)^{5} g(\alpha) e(-\alpha \phi(n)) d \alpha\right|>\frac{1}{2} \cdot \frac{\phi(n)^{5 / 6}}{(\log n)^{\gamma}}
$$

Define the complex numbers $\eta_{n}$ by taking $\eta_{n}=0$ when $n \notin \mathcal{Z}(N)$, and when $n \in \mathcal{Z}(N)$ by means of the equation

$$
\left|\int_{\mathfrak{m}} f(\alpha)^{5} g(\alpha) e(-\alpha \phi(n)) d \alpha\right|=\eta_{n} \int_{\mathfrak{m}} f(\alpha)^{5} g(\alpha) e(-\alpha \phi(n)) d \alpha .
$$


Then it follows from (3.3) that

$$
\frac{N^{5 / 3}}{(\log N)^{\gamma}} \operatorname{card}(\mathcal{Z}(N)) \ll \int_{\mathfrak{m}} f(\alpha)^{5} g(\alpha) K(-\alpha) d \alpha,
$$

where $K(\alpha)$ is defined as in (2.6). On applying Schwarz's inequality to (3.4), we find that

$$
\frac{N^{5 / 3}}{(\log N)^{\gamma}} \operatorname{card}(\mathcal{Z}(N)) \ll I_{1}^{1 / 2}\left(\int_{0}^{1}|K(\alpha) f(\alpha) g(\alpha)|^{2} d \alpha\right)^{1 / 2}
$$

where $I_{1}$ is defined as in (2.8). Thus, by (2.9) and Lemma 3.1, we obtain

$$
\frac{N^{5 / 3}}{(\log N)^{\gamma}} \operatorname{card}(\mathcal{Z}(N)) \ll P^{4}(\log P)^{\varepsilon-3 / 2}
$$

The conclusion of Theorem 1.4 follows on noting that $P \asymp N^{2 / 3}$, and summing over dyadic intervals.

The ideas underlying the proof of Theorem 1.4 are susceptible to generalisation, and we illustrate such extensions with a few additional conclusions. We first require some notation. Let $k \geq 2$ be a fixed integer, and denote by $R_{1}(n)$ the number of representations of the integer $n$ in the form

$$
n=x^{2}+y_{1}^{4}+y_{2}^{4}+y_{3}^{4}+y_{4}^{4}+z^{k},
$$

with $x, y_{i}, z \in \mathbb{N}$. Let $R_{2}(n)$ denote the corresponding number of representations of $n$ in the form

$$
n=x_{1}^{4}+x_{2}^{4}+\ldots+x_{10}^{4},
$$

with $x_{i} \in \mathbb{N}$, and let $R_{3}(n)$ denote the number of representations of $n$ as

$$
n=x_{1}^{3}+x_{2}^{3}+x_{3}^{3}+x_{4}^{3}+y_{1}^{4}+y_{2}^{4},
$$

with $x_{i}, y_{j} \in \mathbb{N}$. When $l$ is a natural number, write

$$
S_{l}(q, a)=\sum_{r=1}^{q} e\left(a r^{l} / q\right)
$$

and

$$
\begin{gathered}
U_{1}(q, a)=q^{-6} S_{2}(q, a) S_{4}(q, a)^{4} S_{k}(q, a), \\
U_{2}(q, a)=q^{-10} S_{4}(q, a)^{10}, \quad U_{3}(q, a)=q^{-6} S_{3}(q, a)^{4} S_{4}(q, a)^{2} .
\end{gathered}
$$

Now define the singular series

$$
\mathfrak{S}_{i}(n)=\sum_{q=1}^{\infty} \sum_{\substack{a=1 \\(a, q)=1}}^{q} U_{i}(q, a) e(-a n / q) \quad(i=1,2,3) .
$$

The methods of Chapters 2 and 4 of Vaughan [19] may be used to show that for $i=1,2,3$ one has $0 \leq \mathfrak{S}_{i}(n) \ll 1$ uniformly in $n$, and indeed 
that $\mathfrak{S}_{i}(n) \gg 1$ whenever $n$ lies in the union of a non-empty collection of congruence classes. Indeed, when $i=3$ one has $\mathfrak{S}_{3}(n) \gg 1$ for all $n$. Finally, when $\phi$ is an integral polynomial and $\gamma$ is a fixed positive number, denote by $\mathcal{E}_{\phi}^{(i)}(N ; \gamma)$ the number of integers $n$ with $1 \leq n \leq N$ for which $\phi(n)$ is positive, and

$$
\left|R_{i}(\phi(n))-R_{i}^{*}(\phi(n))\right|>\frac{\phi(n)^{e_{i}}}{(\log n)^{\gamma}},
$$

where we write $e_{1}=1 / 2+1 / k, e_{2}=3 / 2, e_{3}=5 / 6$, and

$$
\begin{aligned}
& R_{1}^{*}(m)=\frac{\Gamma(3 / 2) \Gamma(5 / 4)^{4} \Gamma(1+1 / k)}{\Gamma(3 / 2+1 / k)} \mathfrak{S}_{1}(m) m^{e_{1}}, \\
& R_{2}^{*}(m)=\frac{\Gamma(5 / 4)^{10}}{\Gamma(5 / 2)} \mathfrak{S}_{2}(m) m^{e_{2}}, \\
& R_{3}^{*}(m)=\frac{\Gamma(4 / 3)^{4} \Gamma(5 / 4)^{2}}{\Gamma(11 / 6)} \mathfrak{S}_{3}(m) m^{e_{3}} .
\end{aligned}
$$

Theorem 3.2. Let $\phi$ be an integral quadratic polynomial with positive leading coefficient. Then, whenever $\gamma>0$ is sufficiently small, for $i=1,2,3$ there is a positive number $\delta$, depending at most on $\gamma$, such that

$$
\mathcal{E}_{\phi}^{(i)}(N ; \gamma) \ll N(\log N)^{-\delta}
$$

Here, the implicit constants in Vinogradov's notation may depend on $\phi, \gamma$ and $\delta$.

Proof. Let $\phi$ be as in the statement of the theorem, and let $\gamma>0$. Define $\mathcal{Z}_{i}(N)$ to be the set of all integers $n$ with $N / 2<n \leq N$ for which (3.5) holds. The goal is to establish the inequality $\operatorname{card}\left(\mathcal{Z}_{i}(N)\right) \ll_{\phi} N(\log N)^{-\delta}$, for sufficiently small values of $\gamma$ and $\delta$. The theorem then follows by summing over dyadic intervals.

When $l$ is a natural number, write $P_{l}=\phi(N)^{1 / l}$ and

$$
f_{l}(\alpha)=\sum_{x \leq P_{l}} e\left(\alpha x^{l}\right) .
$$

Then, for each integer $n$ with $N / 2<n \leq N$ one has

$$
R_{i}(\phi(n))=\int_{0}^{1} \mathcal{F}_{i}(\alpha) f_{4}(\alpha)^{2} e(-\alpha \phi(n)) d \alpha,
$$

where we write

$$
\mathcal{F}_{1}(\alpha)=f_{2}(\alpha) f_{4}(\alpha)^{2} f_{k}(\alpha), \quad \mathcal{F}_{2}(\alpha)=f_{4}(\alpha)^{8}, \quad \mathcal{F}_{3}(\alpha)=f_{3}(\alpha)^{4} .
$$

In the interest of brevity we write $K_{1}=\max \{4, k\}, K_{2}=K_{3}=4$, and then define, for $i=1,2,3$, the major arcs $\mathfrak{M}_{i}$ as the union of the intervals

$$
\mathfrak{M}_{i}(q, a)=\left\{\alpha \in[0,1):|q \alpha-a| \leq P_{K_{i}}\left(2 K_{i} \phi(N)\right)^{-1}\right\},
$$


with $0 \leq a \leq q \leq\left(2 K_{i}\right)^{-1} P_{K_{i}}$ and $(a, q)=1$. Also, we write $\mathfrak{m}_{i}=[0,1) \backslash \mathfrak{M}_{i}$. Then one may apply the methods of Chapters 2 and 4 of Vaughan [19] to establish that there is a positive number $\tau$ such that whenever $N / 2<n \leq N$, one has

$$
\int_{\mathfrak{M}_{i}} \mathcal{F}_{i}(\alpha) f_{4}(\alpha)^{2} e(-\alpha \phi(n)) d \alpha=R_{i}^{*}(\phi(n))+O\left(\phi(n)^{e_{i}-\tau}\right) .
$$

Thus we may conclude that for $n \in \mathcal{Z}_{i}(N)$,

$$
\left|\int_{\mathfrak{m}_{i}} \mathcal{F}_{i}(\alpha) f_{4}(\alpha)^{2} e(-\alpha \phi(n)) d \alpha\right|>\frac{1}{2} R_{i}^{*}(\phi(n))(\log n)^{-\gamma} .
$$

Define the complex numbers $\eta_{n}=\eta_{n, i}$ by taking $\eta_{n}=0$ when $n \notin \mathcal{Z}_{i}(N)$, and when $n \in \mathcal{Z}_{i}(N)$, by means of the equation

$$
\left|\int_{\mathfrak{m}_{i}} \mathcal{F}_{i}(\alpha) f_{4}(\alpha)^{2} e(-\alpha \phi(n)) d \alpha\right|=\eta_{n} \int_{\mathfrak{m}_{i}} \mathcal{F}_{i}(\alpha) f_{4}(\alpha)^{2} e(-\alpha \phi(n)) d \alpha .
$$

We thus obtain from (3.6) the relation

$$
\frac{N^{2 e_{i}}}{(\log N)^{\gamma}} \operatorname{card}\left(\mathcal{Z}_{i}(N)\right) \ll \int_{\mathfrak{m}_{i}} \mathcal{F}_{i}(\alpha) f_{4}(\alpha)^{2} K(-\alpha) d \alpha,
$$

where $K(\alpha)$ is defined as in (2.6). On applying Schwarz's inequality to (3.7), we obtain

$$
\begin{aligned}
& \frac{N^{2 e_{i}}}{(\log N)^{\gamma}} \operatorname{card}\left(\mathcal{Z}_{i}(N)\right) \\
& \ll\left(\int_{\mathfrak{m}_{i}}\left|\mathcal{F}_{i}(\alpha)\right|^{2} d \alpha\right)^{1 / 2}\left(\int_{0}^{1}\left|K(\alpha) f_{4}(\alpha)^{2}\right|^{2} d \alpha\right)^{1 / 2} .
\end{aligned}
$$

In order to complete the proof of the theorem, we must recall some mean value estimates. By considering the underlying diophantine equation, it follows from Lemma 3.1 that

$$
\int_{0}^{1}\left|K(\alpha) f_{4}(\alpha)^{2}\right|^{2} d \alpha \ll N^{2}(\log N)^{\varepsilon} .
$$

Moreover, by employing in the respective cases the methods of Chapter 2 of Vaughan [19] combined with Lemma 3.1 and the methods of Vaughan [17], [18], one has

$$
\int_{\mathfrak{m}_{i}}\left|\mathcal{F}_{i}(\alpha)\right|^{2} d \alpha \ll N^{4 e_{i}}(\log N)^{-3 \tau},
$$

for a suitable positive number $\tau$. Consequently, on combining (3.8)-(3.10) we arrive at the conclusion

$$
\operatorname{card}\left(\mathcal{Z}_{i}(N)\right) \ll N(\log N)^{\gamma-\tau},
$$

which suffices to establish the theorem. 
4. Waring's problem for larger exponents. We conclude this paper with the proof of Theorem 1.5. Here we work slightly harder than is necessary for the application at hand, since we intend to apply some of the associated apparatus in future work connected with this topic. We note that the error term implicit in Theorem 1.5 could certainly be reduced with additional effort, but that we have chosen brevity over precision. We begin by recording some notation. Let $\phi_{l}$ be an integral polynomial of degree $l \geq 2$ with positive leading coefficient, and suppose that $k$ is sufficiently large. We take $N$ to be a large real number. Suppose that $\left(\widetilde{\eta}_{n}\right)$ is a sequence of complex numbers with $\left|\widetilde{\eta}_{n}\right| \leq 1$ for each $n$. We define the exponential sums

$$
\widetilde{K}_{l}(\alpha)=\sum_{N / 2<n \leq N} \widetilde{\eta}_{n} e\left(\alpha \phi_{l}(n)\right) \quad \text { and } \quad \mathcal{K}_{l}(\alpha)=\sum_{N / 2<n \leq N} e\left(\alpha \phi_{l}(n)\right) .
$$

Our argument is facilitated by the following technical lemma.

Lemma 4.1. Suppose that $l$ is an integer with $l \geq 2$. Let $\left(a_{n}\right),\left(b_{n}\right)$ and $\left(c_{n}\right)$ be sequences of non-negative real numbers satisfying the condition that when $n$ is sufficiently large in terms of $N$, one has $a_{n}=b_{n}=c_{n}=0$. Define

$$
\mathcal{F}(\alpha)=\sum_{n \in \mathbb{N}} a_{n} e(n \alpha), \quad \mathcal{G}(\alpha)=\sum_{n \in \mathbb{N}} b_{n} e(n \alpha), \quad \mathcal{H}(\alpha)=\sum_{n \in \mathbb{N}} c_{n} e(n \alpha),
$$

and suppose that

$$
\begin{aligned}
& \int_{0}^{1}|\mathcal{F}(\alpha)|^{2} d \alpha \ll \mathcal{F}(0)^{2} N^{\varepsilon-l}, \\
& \int_{0}^{1}|\mathcal{G}(\alpha)|^{2 l} d \alpha \ll \mathcal{G}(0)^{2 l} N^{\varepsilon-l+l 2^{-l}} .
\end{aligned}
$$

Also, when $l>2$, suppose that

$$
\int_{0}^{1}|\mathcal{H}(\alpha)|^{2 l /(l-2)} d \alpha \ll \mathcal{H}(0)^{2 l /(l-2)} N^{\varepsilon-l} .
$$

Then one has

$$
\int_{0}^{1}\left|\widetilde{K}_{l}(\alpha) \mathcal{F}(\alpha) \mathcal{G}(\alpha)^{2} \mathcal{H}(\alpha)\right| d \alpha \ll N^{-l+1+\varepsilon} \mathcal{F}(0) \mathcal{G}(0)^{2} \mathcal{H}(0) .
$$

Proof. We begin by estimating the auxiliary integral

$$
I_{1}=\int_{0}^{1}\left|\widetilde{K}_{l}(\alpha) \mathcal{G}(\alpha)^{2} \mathcal{H}(\alpha)\right|^{2} d \alpha
$$


Observe that by orthogonality, the integral $I_{1}$ counts the solutions of the diophantine equation

$$
n_{1}+m_{1}+m_{2}+\phi_{l}\left(z_{1}\right)=n_{2}+m_{3}+m_{4}+\phi_{l}\left(z_{2}\right),
$$

with $N / 2<z_{1}, z_{2} \leq N$, and with each solution $\mathbf{n}, \mathbf{m}, \mathbf{z}$ counted with weight at most

$$
b_{m_{1}} b_{m_{2}} b_{m_{3}} b_{m_{4}} c_{n_{1}} c_{n_{2}}\left|\widetilde{\eta}_{z_{1}} \widetilde{\eta}_{z_{2}}\right| \text {. }
$$

On applying the available bound $\left|\widetilde{\eta}_{z}\right| \leq 1$ for each $z$, we therefore deduce that

$$
I_{1} \leq \int_{0}^{1}\left|\mathcal{K}_{l}(\alpha) \mathcal{G}(\alpha)^{2} \mathcal{H}(\alpha)\right|^{2} d \alpha .
$$

We now apply the Hardy-Littlewood method. When $Q$ is a positive real number, we define the major $\operatorname{arcs} \mathfrak{M}(Q)$ to be the union of the intervals

$$
\mathfrak{M}(q, a ; Q)=\left\{\alpha \in[0,1):|q \alpha-a| \leq Q N^{-l}\right\},
$$

with $0 \leq a \leq q \leq Q$ and $(a, q)=1$. For the sake of concision, we write

$$
\mathfrak{M}=\mathfrak{M}\left(N^{1 / 2}\right), \quad \mathfrak{P}=\mathfrak{M}\left(N^{l 2^{-l}}\right), \quad \mathfrak{m}=[0,1) \backslash \mathfrak{M}, \quad \mathfrak{p}=[0,1) \backslash \mathfrak{P} .
$$

By Weyl's inequality (see, for example, Lemma 2.4 of Vaughan [19]), one has

$$
\sup _{\alpha \in \mathfrak{m}}\left|\mathcal{K}_{l}(\alpha)\right| \ll N^{1-2^{-l}+\varepsilon} .
$$

On the other hand, it follows from Theorems 7.1-7.3 of Vaughan [19] that whenever $\alpha \in \mathfrak{M}\left(q, a ; N^{1 / 2}\right) \subseteq \mathfrak{M}$, one has

$$
\begin{aligned}
\left|\mathcal{K}_{l}(\alpha)\right| & \ll N^{1+\varepsilon}\left(q+\phi_{l}(N)|q \alpha-a|\right)^{-1 / l}+\left(q+\phi_{l}(N)|q \alpha-a|\right) \\
& \ll N^{1+\varepsilon}\left(q+N^{l}|q \alpha-a|\right)^{-1 / l}+N^{1 / 2} .
\end{aligned}
$$

In particular, we deduce from (4.6)-(4.8) that whenever $l \geq 2$, one has

$$
\sup _{\alpha \in \mathfrak{p}}\left|\mathcal{K}_{l}(\alpha)\right| \ll N^{1-2^{-l}+\varepsilon} .
$$

Define the function $\Delta(\alpha)$ on $[0,1)$ by taking $\Delta(\alpha)=\left(q+N^{l}|q \alpha-a|\right)^{-1}$ when $\alpha \in \mathfrak{M}\left(q, a ; N^{l 2^{-l}}\right) \subseteq \mathfrak{P}$, and by defining $\Delta(\alpha)=0$ otherwise. Then we conclude from (4.8) and (4.9) that uniformly for $\alpha \in[0,1)$, one has

$$
\left|\mathcal{K}_{l}(\alpha)\right| \ll N^{1+\varepsilon} \Delta(\alpha)^{1 / l}+N^{1-2^{-l}+\varepsilon} .
$$


On substituting (4.10) into (4.5), we obtain

$$
I_{1} \ll I_{2}+I_{3},
$$

where

$$
I_{2}=N^{2+\varepsilon} \int_{0}^{1}\left|\Delta(\alpha)^{1 / l} \mathcal{G}(\alpha)^{2} \mathcal{H}(\alpha)\right|^{2} d \alpha
$$

and

$$
I_{3}=N^{2-2^{1-l}+\varepsilon} \int_{0}^{1}\left|\mathcal{G}(\alpha)^{2} \mathcal{H}(\alpha)\right|^{2} d \alpha .
$$

Suppose temporarily that $l \geq 3$. Then on applying Hölder's inequality to (4.13) in combination with the hypotheses (4.2) and (4.3), we obtain

$$
\begin{aligned}
I_{3} & \leq N^{2-2^{1-l}+\varepsilon}\left(\int_{0}^{1}|\mathcal{G}(\alpha)|^{2 l} d \alpha\right)^{2 / l}\left(\int_{0}^{1}|\mathcal{H}(\alpha)|^{2 l /(l-2)} d \alpha\right)^{1-2 / l} \\
& \ll \mathcal{G}(0)^{4} \mathcal{H}(0)^{2} N^{2-2^{1-l}+\varepsilon}\left(N^{\varepsilon-l+l 2^{-l}}\right)^{2 / l}\left(N^{\varepsilon-l}\right)^{1-2 / l} \\
& \ll \mathcal{G}(0)^{4} \mathcal{H}(0)^{2} N^{2-l+\varepsilon} .
\end{aligned}
$$

On the other hand, an application of Hölder's inequality to (4.12) yields

$$
I_{2} \leq N^{2+\varepsilon} I_{4}^{2 / l}\left(\int_{0}^{1}|\mathcal{H}(\alpha)|^{2 l /(l-2)} d \alpha\right)^{1-2 / l},
$$

where

$$
I_{4}=\int_{\mathfrak{P}} \Delta(\alpha)|\mathcal{G}(\alpha)|^{2 l} d \alpha .
$$

But on recalling (4.6), it follows from Lemma 2 of Brüdern [3] that

$$
I_{4} \ll N^{\varepsilon-l}\left(N^{l 2^{-l}} \int_{0}^{1}|\mathcal{G}(\alpha)|^{2 l} d \alpha+|\mathcal{G}(0)|^{2 l}\right) .
$$

We note here that the estimate (4.16) remains valid also when $l=2$. In view of the hypotheses (4.2) and (4.3), we therefore deduce from (4.15) that

$$
\begin{aligned}
I_{2} & \ll \mathcal{G}(0)^{4} \mathcal{H}(0)^{2} N^{2+\varepsilon}\left(N^{\varepsilon-2 l+l 2^{1-l}}+N^{\varepsilon-l}\right)^{2 / l}\left(N^{\varepsilon-l}\right)^{1-2 / l} \\
& \ll \mathcal{G}(0)^{4} \mathcal{H}(0)^{2} N^{2-l+\varepsilon} .
\end{aligned}
$$

When $l=2$, meanwhile, the estimate (4.14) follows from the hypothesis (4.2) via the trivial estimate

$$
I_{3} \leq N^{2-2^{1-l}+\varepsilon} \mathcal{H}(0)^{2} \int_{0}^{1}|\mathcal{G}(\alpha)|^{4} d \alpha,
$$


and the estimate (4.17) follows similarly from (4.2), (4.16), and the trivial estimate

$$
I_{2} \ll N^{2+\varepsilon} \mathcal{H}(0)^{2} \int_{0}^{1} \Delta(\alpha)|\mathcal{G}(\alpha)|^{4} d \alpha .
$$

Thus, on collecting together $(4.11),(4.14)$ and (4.17), we conclude that when $l \geq 2$, one has

$$
I_{1} \ll \mathcal{G}(0)^{4} \mathcal{H}(0)^{2} N^{2-l+\varepsilon} .
$$

The proof of the lemma is completed on applying Schwarz's inequality to obtain

$$
\int_{0}^{1}\left|\widetilde{K}_{l}(\alpha) \mathcal{F}(\alpha) \mathcal{G}(\alpha)^{2} \mathcal{H}(\alpha)\right| d \alpha \leq I_{1}^{1 / 2}\left(\int_{0}^{1}|\mathcal{F}(\alpha)|^{2} d \alpha\right)^{1 / 2},
$$

where $I_{1}$ is defined as in (4.4), and then substituting from (4.1) and (4.18) to conclude that

$$
\begin{aligned}
\int_{0}^{1}\left|\widetilde{K}_{l}(\alpha) \mathcal{F}(\alpha) \mathcal{G}(\alpha)^{2} \mathcal{H}(\alpha)\right| d \alpha & \ll\left(\mathcal{G}(0)^{4} \mathcal{H}(0)^{2} N^{2-l+\varepsilon}\right)^{1 / 2}\left(\mathcal{F}(0)^{2} N^{\varepsilon-l}\right)^{1 / 2} \\
& \ll \mathcal{F}(0) \mathcal{G}(0)^{2} \mathcal{H}(0) N^{1-l+\varepsilon} .
\end{aligned}
$$

Before proceeding to the main body of our argument, we pause to record some auxiliary mean value estimates. In this context, we define $P$ by means of the relation $P^{k}=\phi_{l}(N)$, and then write

$$
h(\alpha)=\sum_{x \leq P} e\left(\alpha x^{k}\right)
$$

Lemma 4.2. Define the natural numbers $t, u, v, w$ by

$$
t=k\left\lceil\frac{1}{2} k(\log k+\log \log k+1)\right\rceil, \quad u=3 k^{2}, \quad v=k^{2}
$$

and

$$
w=k\left\lceil\frac{1}{2}(1-2 / l) k(\log k+\log \log k+1)\right\rceil+k\lceil 3(1-2 / l) k\rceil+k .
$$

Then

$$
\begin{aligned}
& \int_{0}^{1}|h(\alpha)|^{2(t+u)} d \alpha \ll P^{2(t+u)-k}, \\
& \int_{0}^{1}|h(\alpha)|^{2 v l} d \alpha \ll P^{2 v l-k\left(1-2^{-l}\right)},
\end{aligned}
$$

and when $l>2$, one has also

$$
\int_{0}^{1}|h(\alpha)|^{2 w l /(l-2)} d \alpha \ll P^{2 w l /(l-2)-k} .
$$


Proof. We recall that by employing the version of Vinogradov's mean value theorem due to Wooley [20] within Theorem 1 of Ford [7] (see also Lemma 5.2 of Ford [7]), one finds that for each natural number $r$ with $1 \leq r \leq k(\log k-\log \log k)$,

$$
\int_{0}^{1}|h(\alpha)|^{2 r k} d \alpha \ll P^{2 r k-k+\Delta_{r}}
$$

where

$$
\Delta_{r}=k e^{1-2 r / k} .
$$

The estimate (4.20) is immediate from (4.22) and (4.23), since

$$
\Delta_{v l / k}=k e^{1-2 l}<k 2^{-l} .
$$

We note also that $w l /(l-2) \geq t+u$, and so, possibly by employing the trivial estimate $|h(\alpha)| \leq P$, one finds that (4.21) is an immediate consequence of (4.19).

We concentrate now on establishing the bound (4.19). Here we again employ the Hardy-Littlewood method. In the notation introduced in the proof of Lemma 4.1, we take as our Hardy-Littlewood dissection the set of $\operatorname{arcs} \mathfrak{N}=\mathfrak{M}(P /(2 k))$ and $\mathfrak{n}=[0,1) \backslash \mathfrak{N}$. Then by Vinogradov's version of Weyl's inequality (see, for example, Theorem 5.3 of Vaughan [19]), one has

$$
\sup _{\alpha \in \mathfrak{n}}|h(\alpha)| \ll P^{1-\sigma(k)},
$$

where $\sigma(k)^{-1}=5 k^{2} \log k$. But by (4.22) and (4.23), one has

$$
\int_{0}^{1}|h(\alpha)|^{2 t} d \alpha \ll P^{2 t-k+\Delta_{t / k}}
$$

where

$$
\Delta_{t / k} \leq k \exp (1-(\log k+\log \log k+1))=1 / \log k .
$$

Thus

$$
\int_{\mathfrak{n}}|h(\alpha)|^{2(t+u)} d \alpha \leq\left(\sup _{\alpha \in \mathfrak{n}}|h(\alpha)|\right)^{2 u} \int_{0}^{1}|h(\alpha)|^{2 t} d \alpha \ll P^{2(t+u)-k-\delta},
$$

where

$$
\delta=2 u \sigma(k)-1 / \log k=1 /(5 \log k)>0 .
$$

On the other hand, the methods of Chapter 4 of Vaughan [19] show that whenever $s \geq \max \{5, k+1\}$, then one has

$$
\int_{\mathfrak{N}}|h(\alpha)|^{s} d \alpha \ll P^{s-k}
$$


We therefore conclude from (4.25) that

$$
\int_{0}^{1}|h(\alpha)|^{2(t+u)} d \alpha=\int_{\mathfrak{N}}|h(\alpha)|^{2(t+u)} d \alpha+\int_{\mathfrak{n}}|h(\alpha)|^{2(t+u)} d \alpha \ll P^{2(t+u)-k},
$$

and this completes the proof of the lemma.

For the application of interest to us here, we draw the following consequence of Lemma 4.2 .

Corollary. Suppose that $l$ is a natural number with $l \geq 2$. Write

$$
s_{1}=k[(1-1 / l) k(\log k+\log \log k+20)] .
$$

Then we have the upper bound

$$
\int_{0}^{1}\left|\widetilde{K}_{l}(\alpha) h(\alpha)^{s_{1}}\right| d \alpha \ll P^{s_{1}} N^{-l+1+\varepsilon} .
$$

Proof. We apply Lemma 4.1 with

$$
\mathcal{F}(\alpha)=h(\alpha)^{t+u}, \quad \mathcal{G}(\alpha)=h(\alpha)^{v}, \quad \mathcal{H}(\alpha)=h(\alpha)^{w} .
$$

On noting that $P^{k} \asymp N^{l}$, we find that the conclusions (4.19)-(4.21) of Lemma 4.2 imply the validity of the hypotheses (4.1)-(4.3) of Lemma 4.1. On employing the trivial estimate $|h(\alpha)| \leq P$, one therefore finds that the desired conclusion is immediate from Lemma 4.1.

We are now equipped to describe the proof of Theorem 1.5. Write $s=$ $s_{1}+1$, where $s_{1}$ is given by (4.26). Denote by $R(n)$ the number of representations of $n$ as the sum of $s k$ th powers of positive integers. Then a heuristic application of the circle method suggests that whenever $s \geq k+1$, one should have the asymptotic formula

$$
R(n)=\frac{\Gamma(1+1 / k)^{s}}{\Gamma(s / k)} \mathfrak{S}(n) n^{s / k-1}+o\left(n^{s / k-1}\right),
$$

where

$$
\mathfrak{S}(n)=\sum_{q=1}^{\infty} \sum_{\substack{a=1 \\(a, q)=1}}^{q}\left(q^{-1} \sum_{r=1}^{q} e\left(a r^{k} / q\right)\right)^{s} e(-a n / q) .
$$

We note here that Theorem 4.6 of Vaughan [19] shows that whenever $s \geq 4 k$, one has $\mathfrak{S}(n) \gg 1$ uniformly in $n$. We fix a positive number $\gamma$, and denote by $\mathcal{E}(X)$ the number of integers $n$ with $1 \leq n \leq X$ for which $\phi_{l}(n)$ is positive and

$$
\left|R\left(\phi_{l}(n)\right)-\frac{\Gamma(1+1 / k)^{s}}{\Gamma(s / k)} \mathfrak{S}\left(\phi_{l}(n)\right) \phi_{l}(n)^{s / k-1}\right|>\frac{\phi_{l}(n)^{s / k-1}}{(\log n)^{\gamma}} .
$$

Define $\mathcal{Z}(X)$ to be the set of integers $n$ with $X / 2<n \leq X$ for which $\phi_{l}(n)>0$ and the inequality (4.27) holds. Our goal is to show that for some 
positive number $\tau$, one has $\operatorname{card}(\mathcal{Z}(N)) \ll N^{1-\tau}$. By summing over dyadic intervals, we infer from the latter bound that $\mathcal{E}(N)=o(N)$, and when $l \geq 2$, the conclusion of Theorem 1.5 follows immediately from the definitions of $s_{1}$ and $s$.

We begin by noting that for each integer $n$ with $N / 2<n \leq N$, one has

$$
R\left(\phi_{l}(n)\right)=\int_{0}^{1} h(\alpha)^{s} e\left(-\alpha \phi_{l}(n)\right) d \alpha .
$$

Let $\mathfrak{N}$ and $\mathfrak{n}$ be defined as in the proof of Lemma 4.2. Then by Theorem 4.4 of Vaughan [19], there is a positive number $\nu$ such that whenever $N / 2<$ $n \leq N$, one has

$$
\begin{aligned}
\int_{\mathfrak{N}} h(\alpha)^{s} e & \left.-\alpha \phi_{l}(n)\right) d \alpha \\
= & \frac{\Gamma(1+1 / k)^{s}}{\Gamma(s / k)} \mathfrak{S}\left(\phi_{l}(n)\right) \phi_{l}(n)^{s / k-1}+O\left(\phi_{l}(n)^{s / k-1-\nu}\right) .
\end{aligned}
$$

From (4.28) and (4.29), we find that for $n \in \mathcal{Z}(N)$, one has

$$
\left|\int_{\mathfrak{n}} h(\alpha)^{s} e\left(-\alpha \phi_{l}(n)\right) d \alpha\right|>\frac{1}{2} \cdot \frac{\phi_{l}(n)^{s / k-1}}{(\log n)^{\gamma}} .
$$

We next define the complex numbers $\eta_{n}$ by taking $\eta_{n}=0$ for $n \notin \mathcal{Z}(N)$, and when $n \in \mathcal{Z}(N)$ by means of the equation

$$
\left|\int_{\mathfrak{n}} h(\alpha)^{s} e\left(-\alpha \phi_{l}(n)\right) d \alpha\right|=\eta_{n} \int_{\mathfrak{n}} h(\alpha)^{s} e\left(-\alpha \phi_{l}(n)\right) d \alpha .
$$

In view of (4.30), one obtains

$$
\begin{aligned}
\frac{\phi_{l}(N)^{s / k-1}}{(\log N)^{\gamma}} \operatorname{card}(\mathcal{Z}(N)) & \ll \sum_{N / 2<n \leq N} \eta_{n} \int_{\mathfrak{n}} h(\alpha)^{s} e\left(-\alpha \phi_{l}(n)\right) d \alpha \\
& =\int_{\mathfrak{n}} h(\alpha)^{s} K_{l}(-\alpha) d \alpha,
\end{aligned}
$$

where

$$
K_{l}(\alpha)=\sum_{N / 2<n \leq N} \eta_{n} e\left(\alpha \phi_{l}(n)\right) .
$$

A comparison of the exponential sums $K_{l}(\alpha)$ and $\widetilde{K}_{l}(\alpha)$ reveals that, as a consequence of the corollary to Lemma 4.2 , one has

$$
\int_{0}^{1}\left|K_{l}(\alpha) h(\alpha)^{s_{1}}\right| d \alpha \ll P^{s_{1}} N^{-l+1+\varepsilon} .
$$


Thus, on recalling the estimate (4.24), we find that

$$
\begin{aligned}
\int_{\mathfrak{n}} h(\alpha)^{s} K_{l}(-\alpha) d \alpha & \ll\left(\sup _{\alpha \in \mathfrak{n}}|h(\alpha)|\right) \int_{0}^{1}\left|K_{l}(\alpha) h(\alpha)^{s_{1}}\right| d \alpha \\
& \ll P^{s-\sigma(k)} N^{-l+1+\varepsilon} \ll P^{s} N^{1-l-\tau},
\end{aligned}
$$

for a suitable positive number $\tau$. On recalling that $N^{l} \asymp P^{k}$, we therefore conclude from (4.31) that

$$
\frac{P^{s} N^{-l}}{(\log N)^{\gamma}} \operatorname{card}(\mathcal{Z}(N)) \ll P^{s} N^{1-l-\tau},
$$

whence $\operatorname{card}(\mathcal{Z}(N)) \ll N^{1-\tau / 2}$. This completes the proof of Theorem 1.5.

\section{References}

[1] K. D. Boklan, A reduction technique in Waring's problem, I, Acta Arith. 65 (1993), 147-161.

[2] - The asymptotic formula in Waring's problem, Mathematika 41 (1994), 329-347.

[3] J. Brüdern, A problem in additive number theory, Math. Proc. Cambridge Philos. Soc. 103 (1988), 27-33.

[4] J. Brüdern, K. Kawada and T. D. Wooley, Additive representation in thin sequences, I: Waring's problem for cubes, Ann. Sci. École Norm. Sup. (4), in press.

[5] -, 一, 一, Additive representation in thin sequences, II: the binary Goldbach problem, Mathematika, to appear.

[6] J. Brüdern and N. Watt, On Waring's problem for four cubes, Duke Math. J. 77 (1995), 583-606.

[7] K. B. Ford, New estimates for mean values of Weyl sums, Internat. Math. Res. Notices 1995, no. 3, 155-171.

[8] R. Hall and G. Tenenbaum, Divisors, Cambridge Univ. Press, Cambridge, 1988.

[9] G. H. Hardy and J. E. Littlewood, Some problems of "Partitio Numerorum". I, A new solution of Waring's problem, Göttinger Nachr. 1920, 33-54.

[10] D. R. Heath-Brown, The circle method and diagonal cubic forms, Philos. Trans. Roy. Soc. London Ser. A 356 (1998), 673-699.

[11] C. Hooley, On a new technique and its applications to the theory of numbers, Proc. London Math. Soc. (3) 38 (1979), 115-151.

[12] —, On Waring's problem, Acta Math. 157 (1986), 49-97.

[13] -, On Hypothesis $K^{*}$ in Waring's problem, in: Sieve Methods, Exponential Sums and their Applications in Number Theory (Cardiff, 1995), London Math. Soc. Lecture Note Ser. 237, Cambridge Univ. Press, Cambridge, 1997, 175-185.

[14] L.-K. Hua, Additive Theory of Prime Numbers, Amer. Math. Soc., Providence, RI, 1965.

[15] C. M. Skinner and T. D. Wooley, Sums of two k-th powers, J. Reine Angew. Math. 462 (1995), 57-68.

[16] G. Tenenbaum, Fonctions $\Delta$ de Hooley et applications, in: Séminaire de Théorie des Nombres, Paris 1984-85, Progr. Math. 63, Birkhäuser, 1986, 225-239.

[17] R. C. Vaughan, On Waring's problem for cubes, J. Reine Angew. Math. 365 (1986), $122-170$. 
[18] R. C. Vaughan, On Waring's problem for smaller exponents, II, Mathematika 33 (1986), 6-22.

[19] —, The Hardy-Littlewood Method, 2nd ed., Cambridge Univ. Press, Cambridge, 1997.

[20] T. D. Wooley, On Vinogradov's mean value theorem, Mathematika 39 (1992), 379399.

Mathematisches Institut A

Universität Stuttgart

Postfach 801140

D-70511 Stuttgart, Germany

E-mail: bruedern@mathematik.uni-stuttgart.de

Department of Mathematics

University of Michigan

East Hall

525 East University Avenue

Ann Arbor, MI 48109-1109, U.S.A.

E-mail: wooley@math.lsa.umich.edu
Department of Mathematics Faculty of Education Iwate University Morioka 020-8550, Japan E-mail: kawada@iwate-u.ac.jp 